\title{
Hospital Queue Control System using Quick Response Code (QR Code) as Verification of Patient's Arrival
}

\author{
Ridho Hendra Yoga Perdana ${ }^{1}$, Hudiono ${ }^{2}$, Mochammad Taufik ${ }^{3}$, Amalia Eka Rakhmania ${ }^{4}$ \\ Rohman Muhamad Akbar ${ }^{5}$, Zainul Arifin ${ }^{6}$ \\ Electronic Engineering Department \\ State Polytechnic of Malang, Malang, East Java, Indonesia
}

\begin{abstract}
Hospital is an organization that primarily provides services in the form of examination, treatment, medical treatment and other diagnostic measures required by each patient in the limits of the technology and the means provided by the hospital. So that patients get the maximum service, hospitals have to provide the best services, one of the services that are being highlighted is the queue system. This study proposed a queue control system at the hospital using a quick response code (QR Code) as verification of the patient's arrival that serves to speed up the administrative process and provide effective services, to facilitate the patient in terms of the queuing line (registration) at the hospital. The queue control system uses the website as a registration and database repository for patient data. This website has two kinds of display form, as a patient or a doctor. This kind of display is useful to speed up the administrative process, so that registration can be done at any time by the patient, with patient entering/filling data themselves and their complaints or medical records that are automatically available on the website, so that patients do not need to come to the hospital with a medical record. Furthermore, the website will filter the data entered by the patient as the patient's complaints, so that patients can choose the hospital that provides services for patients' illness complaints which are also showing hospital addresses to facilitate patients in finding the location of the hospital. After the administration process on the website, the system will provide a QR Code as a form of verification for patients who have registered, with their data already exists in the database. When the patient arrives at the hospital, the patient only needs to scan the $Q R$ code that already exists, without taking care of the administration back because at the time the system gives a QR Code, indicating that patients already had a hospital destination along with the queue number. It is expected that the queue control system implemented at the hospital using a quick response code ( $Q R$ Code) as verification of the patient's arrival, could speed up the administrative process to be more effective and efficient.
\end{abstract}

Keywords-Queue; hospital; patient; quick response code; registration

\section{INTRODUCTION}

In Indonesia, there is a service that is often used by people to support/serve complaints about public health. This kind of service/support has a primary goal of providing services in the form of examination, treatment, medical treatment, and other diagnostic measures required by each patient in the limits of technology and infrastructure provided by the hospital. The hospital also has rules in serving patients, such as a room or a bed of patients [1][2], the quality of drugs, and the facilities provided [3]. So that patients get the maximum service, hospitals have to provide the best services, one of the services that are being highlighted is the queue system because patients often complain about the length of the queue to the checkout process [4] [5]. In order for hospital operations can run effectively and efficiently, it needs development in the infrastructure field [6]. One is through the implementation of technology.

The development of technology is currently more advanced and has a great advantage, so we need a system that serves to support the advancement of technology that could produce the required information faster and more convenient than the manual method [7]. One example is the Quick Response Code (QR Code), which is a type of code that is able to convey information quickly and get a quick response anyway. Unlike the bar code, which only stores information horizontally, a QR code is able to save the information horizontally and vertically, and therefore automatically a QR code can hold more information than a bar code [8][9]. QR codes have high capacity in the data encoding, which is capable of storing all kinds of data, besides the QR code has a smaller screen than the barcode. This is because the QR code can hold the data horizontally and vertically, so automatically the size of the display of the QR code image can be only $10 \%$ of the size of a barcode. Not only that $\mathrm{QR}$ codes are also resistant to damage, because the QR code is able to correct the error up to $30 \%$. Although some symbol of the QR code is dirty or damaged, the data can still be stored and read [10][11]. Three square-shaped marks in three corners have a function so that the symbol can be read with the same results from any angle throughout 360 degrees [12].

This study proposed a queue control system at the hospital using a quick response code (QR Code) as verification of the patient's arrival. The system provides an effective service to facilitate the patient in terms of administration (queue). The system uses the website as an interface that can be accessed by the user anytime and anywhere. During the registration process line, the patient will enter the data themselves and their complaints on a website, and then they will get their medical records that are automatically stored in the patient account, so the patient does not need to come to the hospital with a medical record. Then the system will filter the data that has been entered by the patient, such as personal data, patient's disease complaints, hospitals that provide services 
for such diseases, as well as hospitals desired address, in order to facilitate the patient in finding the location of the hospital [13]. After the administration process on the website, patient data will be stored in the database and the system will provide a QR Code as verification for patients who have registered. Data stored on the QR Code including hospital lab data that are in accordance with the patient's medical record, which has been filtered by the system along with the queue number, will be automatically processed by the system and generate data stored in the patient's QR Code. When arriving at the hospital, the patient only needs to scan the QR code that has been downloaded, without doing the re-administration.

\section{Methodology}

\section{A. Phase 1; Requirements Planning}

At the stage of making a line control system with Quick Response Code in this hospital is divided into several sections, this section includes several parts:

1) Hospital design: This section describes the workflow tool used in hospitals. It also explains how the doctor is doing the examination process and to provide action on the patient.

2) User: This section describes the concept created for patient registration, booking, arrival verification, as well as the examination of patients by doctors.

3) Relation database: This database describes the concept of the queue control system with Quick Response Code, this design also includes web design and database design.

\section{B. Phase 2; Hospital Design}

Hospital's main purpose for the patient is to obtain medical care, while the hospital workflow process can be seen in Fig. 1.

Fig. 1 is a workflow in hospitals, the first step for the patient is to scan the QR Code to verify the arrival of the patient in order to indicate that the patient has arrived at the hospital. The patients QR code will be scanned with QR Code sensors that are available at the front desk. Further information will appear on the LCD, this information will appear when the patient has already verified for their arrival at the hospital, the information on the LCD is useful to simplify the patient action to get the examination at the hospital. The LCD contains information such as the name of the lab, patient id, queue number, and patient's name. Then the examination process will be done by a doctor, where the doctor will examine the patient while providing certain measures to the patient.

1) System design: This design system is a general description of how the tool works from the queue control system and for the flow can be seen in Fig. 2.

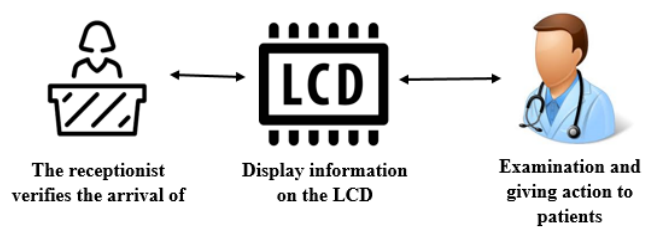

Fig. 1. Work Process Hospital.

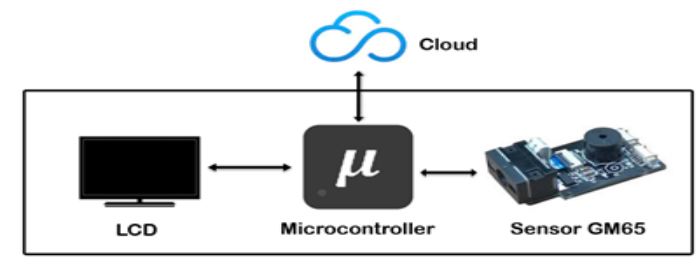

Fig. 2. Machine Operating System.

Fig. 2 shows the design of the tool, how this tool works. First, the GM65 sensor will scan the patient's QR Code to verify the patient's arrival at the hospital, the process of scanning this data is by matching the code that has been obtained by the patient with the code in the database. Second, the microcontroller works to control the process of starting the QR Code scanner, verifying the patient's data, retrieving data in the database, and displaying data on the LCD screen. Third, this cloud function is to connect hardware with software (website) so that the patient data can be stored in the database. Fourth, the LCD will display information to make it easier for patients to go to a certain lab in the hospital.

2) Hardware design: This hardware work process can be seen in Fig. 3.

Fig. 3 descriptions are:

a) Quick Response Code (QR Code): Patients Quick Response Code (QR Code) is used to verify the arrival of patients. The patients can only get a $\mathrm{QR}$ Code after registering on the website; this code will later be scanned to verify the arrival of patients at the hospital.

b) Scan Quick Response Code (QR Code) in hospital: To verify the arrival of patients at the hospital the patient must scan the Quick Response Code (QR Code) at the hospital. This process of scanning data is by matching the code obtained by the patient with the code in the database. If the patient does not register on the website, the patient will not get the Quick Response Code (QR Code). If the code matches, the process will proceed to Arduino Uno.

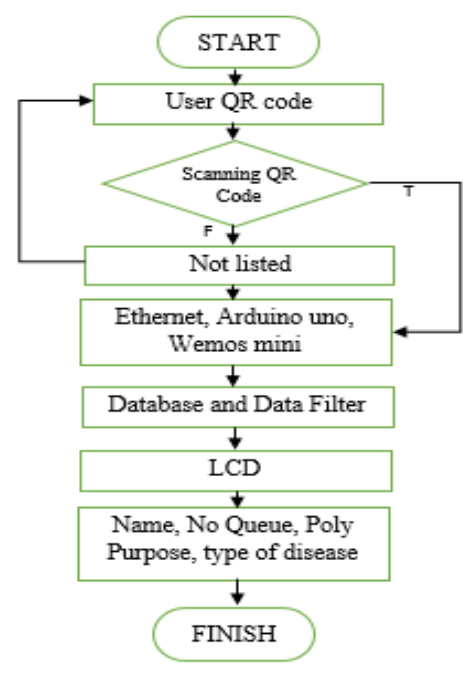

Fig. 3. Tool Design (Hardware). 
c) Ethernet and Arduino Uno: Ethernet is used to connect mobile devices. Which then the mobile device will scan user/patient Quick Response Code (QR Code). Arduino itself is used to match Quick Response Code (QR Code) and filter patient registration data from the database. After filtering, the results of the data selection will be displayed on the Liquid Crystal Display (LCD) screen.

d) Database: This database is used to store patient medical records and patient registration data. This database will be accessible to patients and hospitals. The hospital will access this database to retrieve patient data in accordance with Quick Response Code (QR Code) which is owned by the patient during verification arrival. This database is in the form of a place hosting/server.

e) Wemos Mini: Wemos Mini hardware is used to connect devices to the Internet so that it can access the database that has been provided.

f) Data Filter: This data filter is done by Arduino Uno, because the data filter includes location, disease category, and hospital lab destination. Results of this data filter will be displayed on the Liquid Crystal Display (LCD) screen to facilitate patients in terms of the queue process.

g) Liquid Crystal Display (LCD): Liquid Crystal Display (LCD) is used to display data from the Arduino filter results. The data displayed is in the form of the patient's name, hospital lab destination, and queue number.

Fig. 4 shows the scanning process of the QR Code with the GM65 sensor.

This Quick Response Code is used to verify the arrival of patients in the hospital, namely with GM 65 sensors as shown in Fig. 4.

3) Doctor design: The workflow process of doctors at the hospital can be seen in Fig. 5.

Fig. 5 descriptions are:

a) Login: Doctors must be logged on to the website first before starting the examination of the patient. The login process can be done by entering the username and password that is owned by the doctor.

b) Scan the Patient QR Code: QR Code Scan is done so that doctors can enter the patient's medical record in question and give action to these patients. In giving action, doctors can go through the website or application on Android.

c) Examination and giving action to the patient: The examination will be carried out when the doctor has entered the patient's medical record and gives action to the patient. After that, the doctor will fill in the patient's medical record that has been available on the action form.

Fig. 6 shows the process flow from the doctor. The doctor will $\log$ in first, then the doctor will go into the patient's history to provide follow-up after the examination. Then, the doctor can see the patient's history that is available on the website's features.

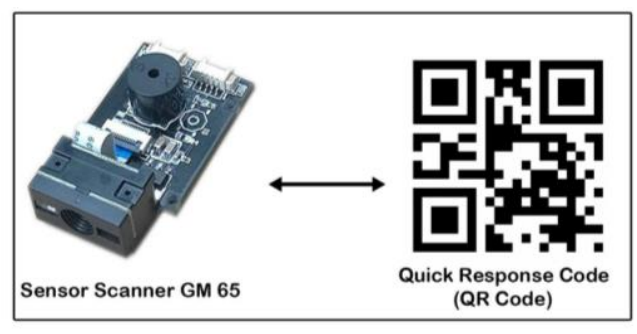

Fig. 4. Doctor Activity Diagram.

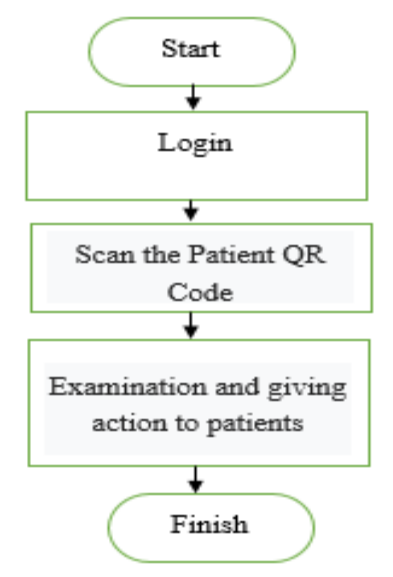

Fig. 5. Doctor Workflow Process.

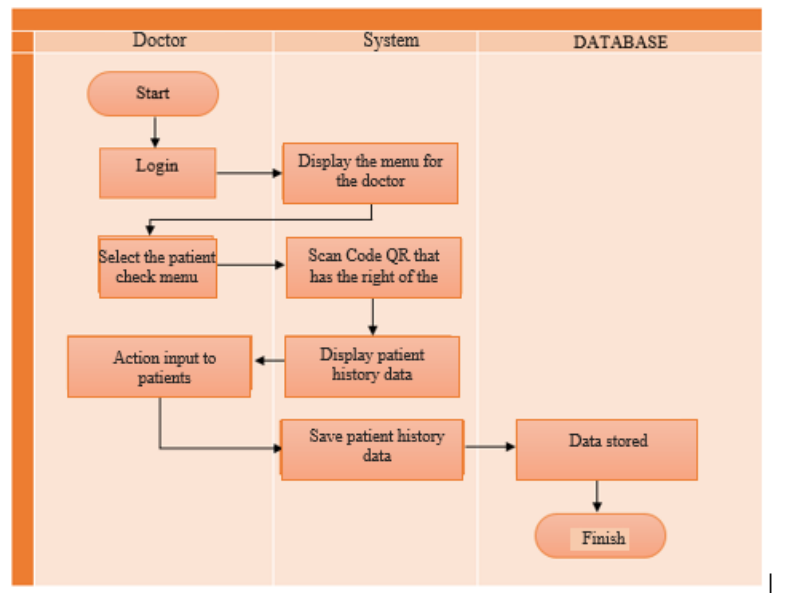

Fig. 6. Doctor Activity Diagram.

\section{Phase 3; user Design}

The input of the queue control system and the patient's workflow process is described in Fig. 7.

Fig. 7 shows a flowchart about the block diagram of the queue control system. The first step is creating an account/login as a patient. Ordering a queue at the hospital can be done by accessing the website and registering a new account to $\log$ in to the website to place an order. Registration form consists of name, address, date of birth, username, email, and password which will be filled by the patient to create a new account, then the patient will enter the website by entering the username and password that has been registered. 


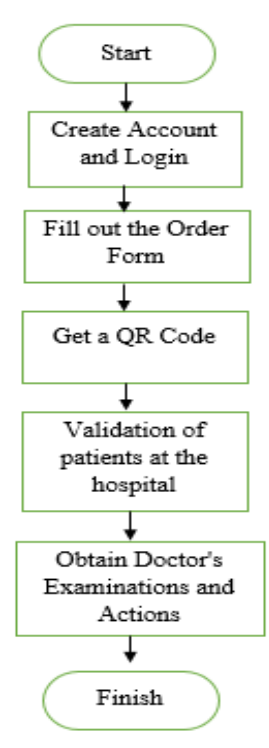

Fig. 7. Queue Control System Block Diagram.

The second step is to fill in the order form. The patient registration form is on the order menu, this order form is filled in by the patient to order queues at the hospital. In the order form, there are fillings for order date, city, hospital, hospital lab, and complaints that will be filled by the patient. After filling out the order form, patients can choose the doctor's schedule that has been provided by the hospital. After pressing order, the patient will get a QR Code. This QR Code will be used to verify arrival at the hospital. This Quick Response Code (QR Code) will be obtained by the patient when the patient has placed an order on the website, the QR code on the website can be downloaded by the patient in a PDF format.

The third step is to verify the arrival at the hospital. To verify the arrival of the patient in the hospital, the patient must scan his QR code (which was obtained during online registration), the QR Code sensor scanner at the hospital. Then on the LCD screen will display hospital lab name, patient id, queue number, and patient name to make it easier for patients to carry out examinations at the hospital.

The fourth step is to get a doctor's examination and action. After the patient verifies the arrival, the patient will undergo an examination and get action from the doctor, and the doctor will scan the patient's QR code to enter the patient's medical record data. Patients can see the examination history of the patient's medical record menu on the website.

Fig. 8 shows the process flow of the user/patient. Patients will register (not already have an account) as well as ordering the queue at the hospital designated by using the website. Patients will enter the data themselves in the provided form and will get a $\mathrm{QR}$ code for arrival verification.

\section{Phase 4; Relation Database Design}

This database is used for data storage on the server. And this database is also used as one of the supporting systems for the queue system at the hospital, and the database workflow can be seen in Fig. 9.

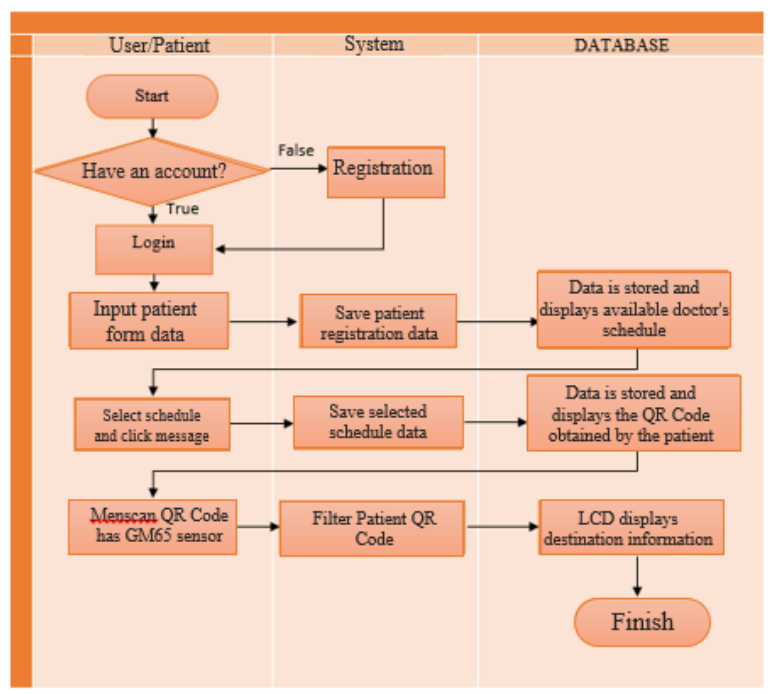

Fig. 8. User Activity Diagram.

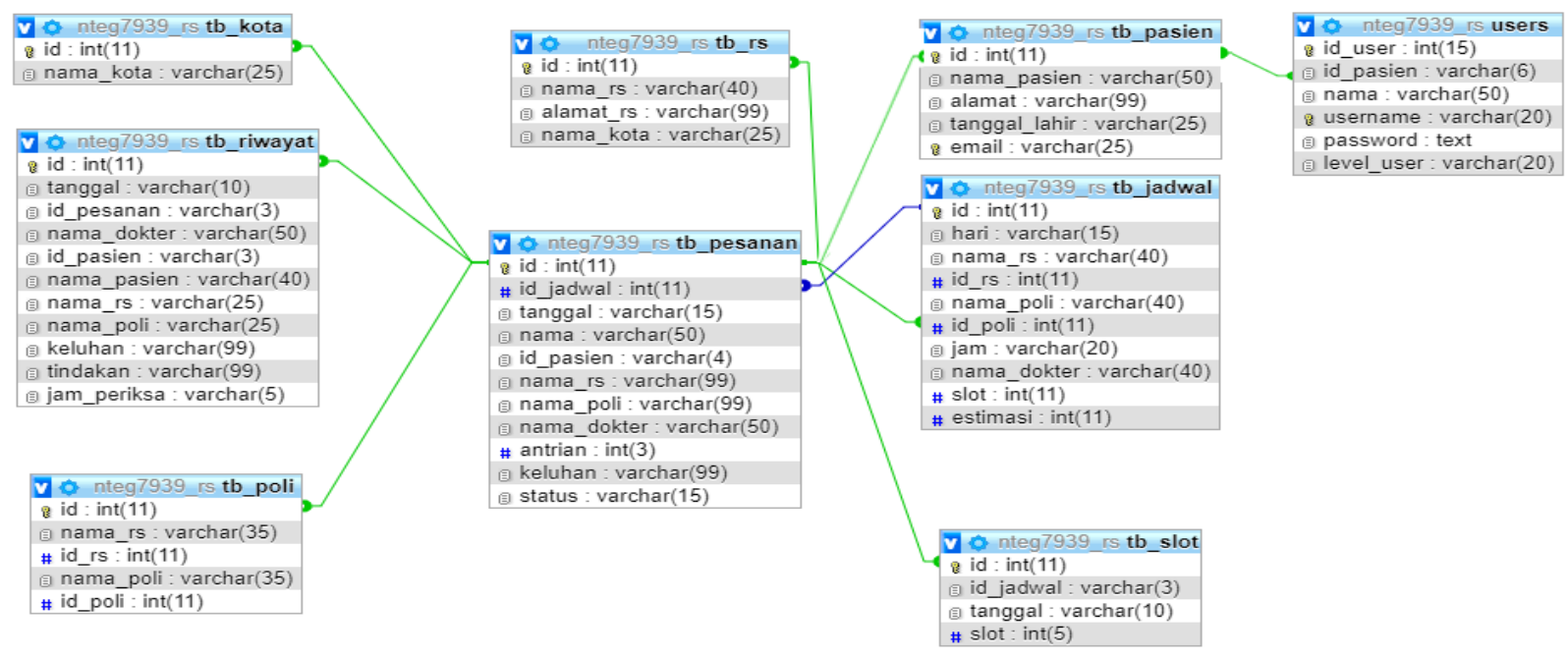

Fig. 9. Relation Database Workflow. 


\section{RESUlT AND DiscUSSION}

\section{A. Hospital}

The results of this hardware are the design that has been made according to the concept. The results can be seen in Fig. 10.

Fig. 10 shows the result of the hardware design, as seen on the image there is 3 hardware, which each hardware will be used in three cities and three different hospitals. The hardware also contains an LCD display that shows information about the hospital lab name, patient name, patient id, and queue number.

1) Doctor: On the Doctor's main page will display the patient's ID to be filled by a doctor by scanning/inputting the QR Code that the patient has. The doctor's main page can be seen in Fig. 11.

On this action form page, there is information about the patient's personal data and there is an action form that will be filled out by the doctor after conducting an examination to the patient, also displaying the doctor's actions which can be seen in Fig. 12.

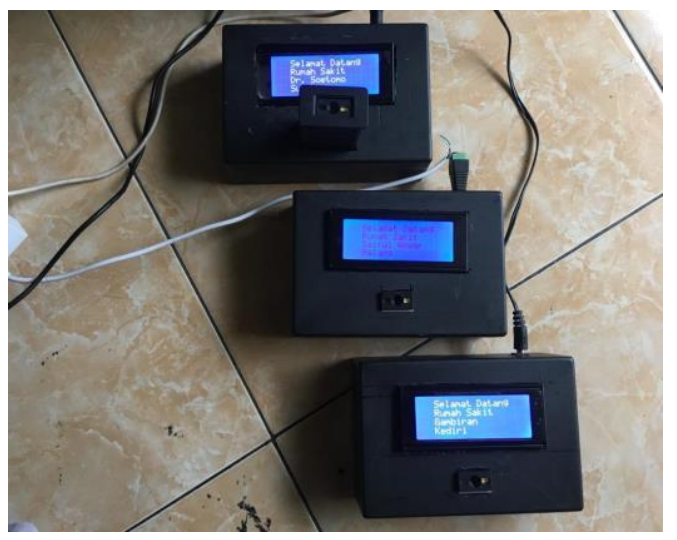

Fig. 10. Hardware Results.

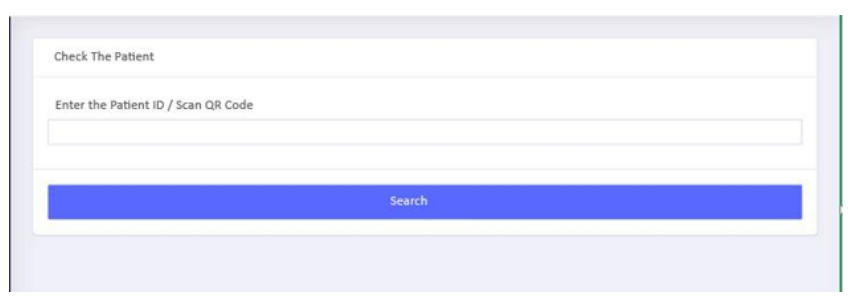

Fig. 11. Doctor Main Page.

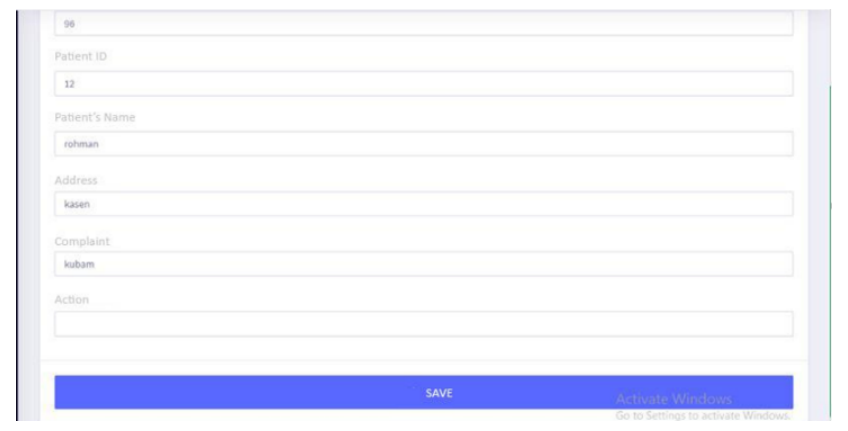

Fig. 12. Action Form Page/Patient Medical Records.
On the examination history page, there is an information display of patients who have been examined by a doctor. In the page, there is also information about the examination date, patient name, complaints, and actions given by the doctor. The check history page can be seen in Fig. 13.

Status menu page displays the names of patients who have registered for the doctor's examination. The status page also contains an information table consisting of the registration date column, order number, queue, patient name, complaints, and patient's presence status. The status menu page can be seen in Fig. 14.

\section{B. User}

This login display is the initial display when the patient or doctor will access the website. In this login display, there are two inputs, namely, "Username" and "Password". To enter/log in the patient/doctor must enter both inputs. Before logging in, patients must first have an account by registering first. And the display of the login page can be seen in Fig. 15.

This registration page is a display when the patient will create a new account. In this registration view there are several inputs such as name, address, username, e-mail, and password where the patient will enter to enter the login page. The appearance of the registration page can be seen in Fig. 16.

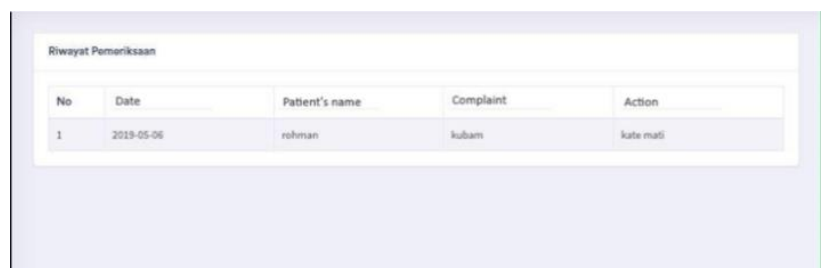

Fig. 13. Examination History Page.

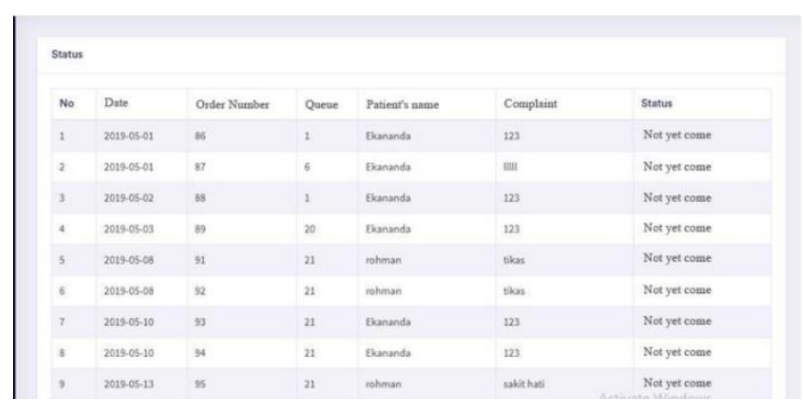

Fig. 14. Status Menu Page.

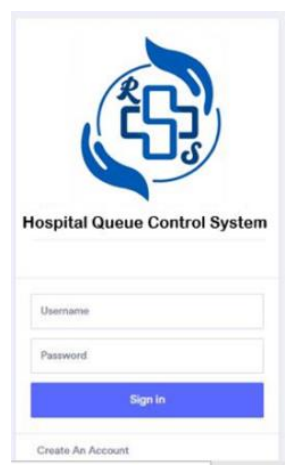

Fig. 15. Login Page. 


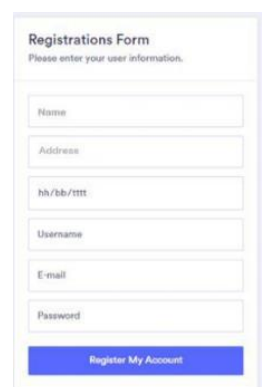

Fig. 16. Registration Page.

This order form page is used by patients to place an order by filling out a form consisting of Date, City, Hospital, hospital lab, and Complaints. On the first form the patient will choose the date first and then the patient chooses the city that the patient wants, where the system will display the hospital according to the city chosen and the hospital lab available at the hospital. After that the patient will enter a complaint that the patient will input according to the complaint of the disease, then the patient clicks on the message to go to the next process. The order form page can be seen in Fig. 17.

The display of the doctor's schedule page shows several names of doctors available at the hospital on that date. This page also provides information to patients to find out which doctor will serve the examination at the hospital. Then the patient clicks the message for the order process. The doctor's schedule page can be seen in Fig. 18.

This page, display the QR Code that will be obtained by the patient to verify arrival at the hospital. This QR Code can be stored by patients in the form of PDF. In this QR Code file, there is information such as the name of the hospital, the name of the hospital lab, order number, queue number and estimated time. This information is to make it easier for patients when doing treatment/examination at the hospital. The QR Code display can be seen in Fig. 19 and fill in the detailed information in the QR Code in PDF format can be seen in Fig. 20.

This map page shows the location of the hospital, which is useful to facilitate patients to go to the hospital, and the display of the maps page is shown in Fig. 21.

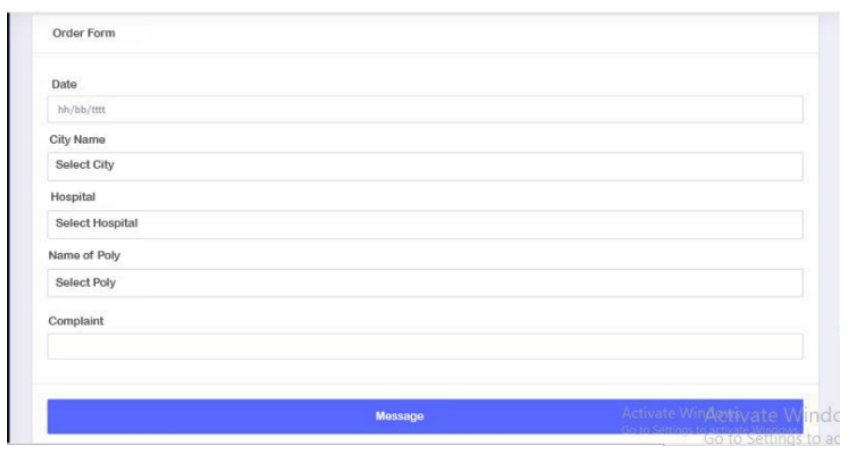

Fig. 17. Order Form.

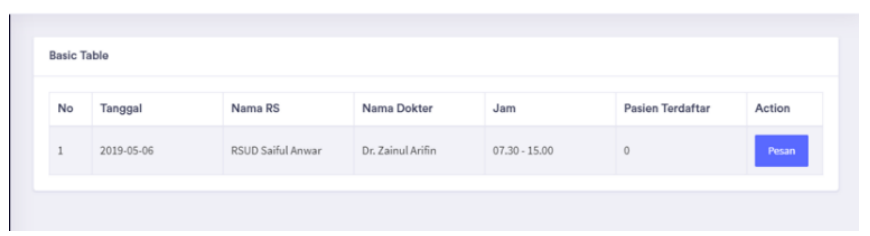

Fig. 18. Doctor Schedule Page.

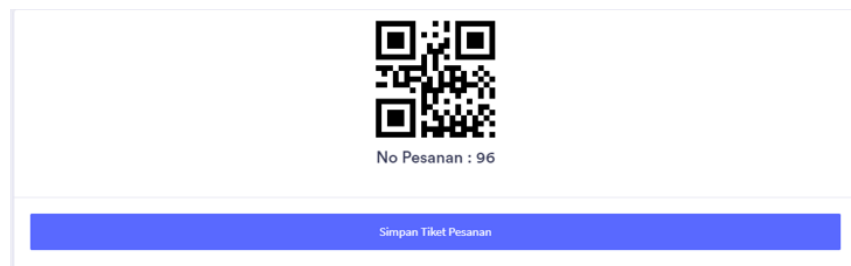

Fig. 19. QR Code Page.

Sistem Antrian Rumah Sakit

$\begin{array}{ll}\text { Rumah Sakit } & \text { RSUD Saiful Anwar } \\ \text { Nama Poli } & \text { Poli Umum } \\ \text { No Pemesanan } & 96 \\ \text { No Antrian } & 21 \\ \text { Diharapkan Datang Sebelum : } 14.10\end{array}$

Fig. 20. Maps in QR Code.

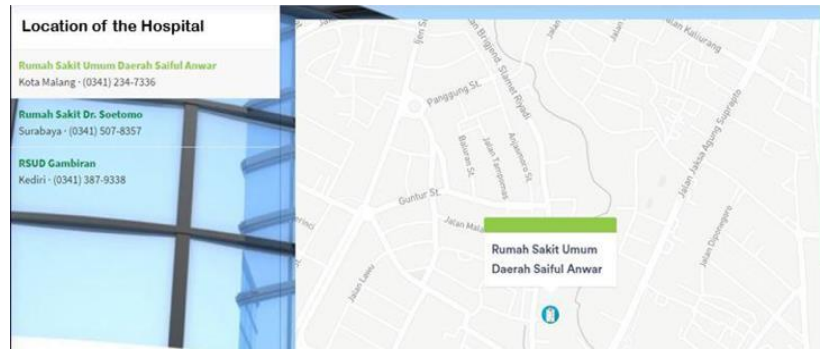

Fig. 21. Maps.

\section{CONCLUSION}

After measuring, testing and analyzing the data, it can be concluded that:

1) By using a website, we can make reservations in the hospital be done anywhere by patients which automatically produces a quick response code (QR Code).

2) For hospital bookings online, it will automatically be integrated so that patients can make hospital bookings listed on the website.

3) The use of integrated queue control systems in hospitals can help manage patients' personal data and medical records.

4) The quick response code (QR Code) can save the order ID that is used to verify the patient's arrival. 


\section{FUTURE WORK}

This queue control system still has advantages and disadvantages, so the suggestions for this queue development system are as follows:

1) To develop the hospital registration or booking system, it can be developed on an Android application with a sound model and patient photos.

2) Need to feature hospital services and facilities when the patient's QR Code has been accumulated on Android.

\section{REFERENCES}

[1] P.-F. Tsai and F.-M. Lin, "An Application of Multi-Attribute Value Theory to Patient-Bed Assignment in Hospital Admission Management: an Empirical Study," J. Healthc. Eng., vol. 5, no. 4, pp. 439-456, 2014.

[2] S. M. Sohrevardi, A. Shafiei, and S. S. Mirzania, "Intravenous Immunoglobulin: A Drug Utilization Review at Shahid Sadoughi Hospital in Yazd," no. 1, 2014.

[3] Ian Brown and Andrew Smale, "Management of Medical technologyCase study of a Major Acute Hospital" 29th Annual International Conference of the IEEE Engineeting in Medicine and Biology Society, 2007.

[4] "Intravenous Immunoglobulin: A Drug Utilization Review at Shahid Sadoughi Hospital in Yazd," no. 1, 2014.

[5] S. M. Mousavi, F. Pourshariati, G. Rajabi, and M. Letafatnejad, "Waiting Time to Receive Healthcare Services and Factors Affecting It : Case Study in a University Hospital," vol. 1, no. 2, 2017.
[6] Q. Su, X. Yao, P. Su, J. Shi, Y. Zhu, and L. Xue, "Hospital registration process reengineering using simulation method," J. Healthc. Eng., vol. 1, no. 1, pp. 67-82, 2010.

[7] A. M. H. Pardede et al., "Framework For Patient Service Queue System For Decision Support System on Smart Health Care," Int. J. Eng. Technol., vol. 7, no. 2.13, pp. 337-340, 2018H. N. Abed and C. Science, "Robust and Secured Image Steganography using LSB and Encryption with QR Code improvement of the amount and security of transmitting information, the secrecy of data method were proposed for information security . In cryptography, the encrypted message was," vol. 9, no. 2, pp. $1-9,2017$.

[8] F. P. Mardiah and M. H. Basri, "The Analysis of Appointment System t o Reduce Outpatient Waiting Time at Indonesia's Public Hospital," Hum. Resour. Manag. Res., vol. 3, no. 1, pp. 27-33, 2013.

[9] Nutchanad Taveerad and Sartid Vongpradhip, "Development of Color QR Code for Increasing Capacity", 11th International Conference on Signal-Image Technology \& Internet-based, 2015, DOI $: 10.1109 /$ SITIS.2015.42.

[10] P. Y. Lin and Y. H. Chen, "High payload secret hiding technology for QR codes," Eurasip J. Image Video Process., vol. 2017, no. 1, 2017.

[11] Muming Li, Peng Cao, Liuping Feng, Lifang Yu, Jianbo Chen and Jing Wang, "The Research of QR code image correction based on image gray feature" First International Conference on Electronics Instrumentation \& Information Systems (EIIS), 2017, DOI : 10.1109/EIIS.2017.8298583.

[12] Vassilya Uzun, "QR-Code Based Hospital Systems for Healtcare in Turkey", IEEE 40th Annual Computer Software and Applications Conference (COMPSAC), volume 2 (2016), DOI: 10.1109/ COMPSAC.2016.173.

[13] D. A. Martillano, R. G. Reyes, I. R. B. Miranda, and K. L. C. Diaz, "Android-Based Smart Power Outlet Switching Device Using ESP8266 Enabled WiFi Module,” vol. 6, no. 1, pp. 61-65, 2018. 